\title{
Automatic Emotions Assessment Using Heart Rate Variability Analysis and 2D Regression Models of Emotions
}

\author{
Sadaf Moharreri ${ }^{1}$, Shahab Rezaei ${ }^{2}$, Nader Jafarnia Dabanloo ${ }^{3}$, Saman Parvaneh ${ }^{3 *}$ \\ ${ }^{1}$ Islamic Azad University, Khomeini Shahr Branch, Isfahan, Iran \\ ${ }^{2}$ Islamic Azad University, Central Tehran Branch, Tehran, Iran \\ ${ }^{3}$ Islamic Azad University, Science and Research Branch, Tehran, Iran \\ ${ }^{*}$ Currently with Philips Research North America, Cambridge, MA, USA
}

\begin{abstract}
Objective assessment of emotions is a challenging task. In this research, a new approach for detection and quantification of four emotions (pleasure, sadness, anger, and joy) is introduced based on the $2 D$ regression models of emotions. ECG signal of 70 healthy female volunteers was recorded while the participants were stimulated by four different colors for five minutes. Induced arousal and valence levels were identified using the Self-Assessment Manikin test. Based on extracted features from Poincare Plot of heart rate series, 1- the label of emotion is determined based on 2-D emotion models using a rulebased approach, and 2- the strength of emotion is calculated in percentages using proposed mathematical formula. The results confirm the findings of previous studies that showed that each color is related to one emotion. The mean square error for estimation of arousal and valence levels were 0.0393 and 0.0536, respectively. In emotion classification, sensitivity of $96.78 \%$, specificity of $99.4 \%$, and the accuracy of $98.75 \%$ were achieved. The proposed method for measuring emotions can be of great help to psychiatrists to identify mental disorders. Using this method, one can measure and express the strength of emotions individually and has a comparative criterion for emotions in different individuals.
\end{abstract}

\section{Introduction}

Emotions are psycho-physiological experiences that affect all aspects of human daily life [1]. It is a complex process that involves many components, such as senses, physical changes, behavior, thoughts, and cognitive responses [1,2]. Different models have been developed using the interaction between these components for assessing emotion, but there is still research opportunities to determine emotions objectively.

Among various physiological biomarkers, heart rate variability (HRV) is a good measure for the assessment of emotional states $[3,4]$ as it has a strong correlation with Autonomic Nervous System that reflects the emotional responses in the human body [5]. Furthermore, a heart rate series recorded using a non-invasive recorder has different responses to the induced emotions [6].

In this article, using extracted features from Poincare plot of heart rate series and 2D regression models of emotions, we proposed a method for labeling and quantifying strength of different emotions.

\section{Data and Method}

\subsection{Data}

ECG (lead II with sampling frequency equal to $200 \mathrm{~Hz}$ ) were recorded from 70 female volunteers (Age: $22.32 \pm 1.65$ ) while the participants were stimulated by four different colors (red, green, blue, and yellow). Each color was presented on a computer screen for five minutes with a randomized order, while participants were seated on a chair. Between each color stimuli, a resting time of two minutes was given to cancel the effects of previous stimulation.

\subsection{Emotion Labels}

After each color stimulus, the participants were asked to consider the nature of the color without any meaning and respond to the Self-Assessment Manikin (SAM) test to assess induced arousal and valence levels. Figures for the valence dimension in SAM range from frowning, unhappy to smiling, happy and the figures range from relaxed, sleepy to excited, and wide-eyed for the arousal dimension

Table 1. The definition of emotions based on arousal and valence
levels
\begin{tabular}{c|c|c} 
Arousal & Valence & Emotion \\
\hline Positive & Positive & Joy \\
\hline Positive & Negative & Anger \\
\hline Negative & Negative & Sadness \\
\hline Negative & Positive & Pleasure
\end{tabular}


[7], Figure 1. The subject can select any of the five figures comprising each scale $(-2,-1,0,+1,+2)$.

Based on arousal and valence levels, emotion for each experiment was labeled as is shown in Table 1. For example, for negative arousal and positive valence, the emotion is called pleasure.

\subsection{Method}

\subsubsection{QRS Detection}

The Pan-Tompkins algorithm was used for QRS detection [8]. Checking by an expert manually, RR interval series were created using corrected QRS peaks.

\subsubsection{Emotion Detection using 2D Regression Model of Emotions}

Moharreri et al. introduced two tree-based arousal and valence models of emotions based on the extracted features from Poincare plot of RR intervals [9]. In this article, we used these models for estimating the arousal and valence levels in each experiment for each subject. For this purpose, first, listed features in Table 2 were extracted. For more details about the extracted features, please refer to [9]. Using the extracted features used in 2D regression models of emotions, arousal and valence levels for each experiment (i.e., recorded signal) were obtained. Based on information in Table 1, four regions/emotions defined based on the level of arousal and valence. Therefore, IFTHEN rules were used to classify these four groups of emotions based on the level of arousal and valence, as follow:

- IF arousal is Positive and valence is Positive THEN emotion is Joy.

- IF arousal is Positive and valence is Negative THEN emotion is Anger.

- IF arousal is Negative and valence is Positive THEN emotion is Pleasure.

- IF arousal is Negative and valence is Negative THEN emotion is Sadness.

\subsubsection{Detection Quantification of Emotions}

After estimating arousal and valence levels, the

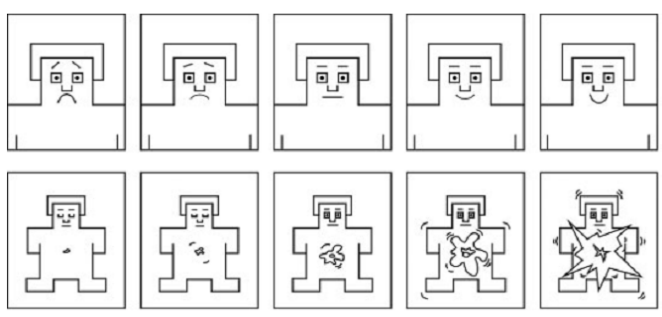

Figure 1. Self-Assessment Manikins (SAM) test following formula was used to determine the strength of emotions in percentage:

$$
\text { Emotion }=\frac{\sqrt{\text { arousal }^{2}+\text { valence }^{2}}}{\sqrt{8}} \times 100
$$

Therefore, it is possible to label the emotion based on Table 1 and show the strength of emotion in percentage using the equation (1). For example, as it is shown in Figure 2, for a case where the arousal level is -2 , and the valence level is +2 detected emotion is a pleasure with the strength of $100 \%$. While for an experiment where the arousal level is -1 and the valence level is +2 detected emotion will be a pleasure with the strength of $79.05 \%$.

\section{Results}

\subsection{Evaluating the Results of Estimating Arousal and Valence Levels}

For testing the estimated results, features extracted from RR series were used as inputs to tree-based models to estimate the value of arousal and valence separately and then, the mean square error for the resulting outputs was calculated as follows:

$M S E=\frac{1}{N} \sum_{i=1}^{N}\left({\left.\text { Real } \text { Value }_{i}-\text { Estimated }_{\text {Value }}\right)^{2}}^{2}\right.$

$\mathrm{N}$ is the number of data, Real Value is the value of the arousal and valence obtained by SAM test, and Estimated Value is the value of arousal and valence obtained by models. The results are shown in Table 3 for arousal tree and valence tree, separately.

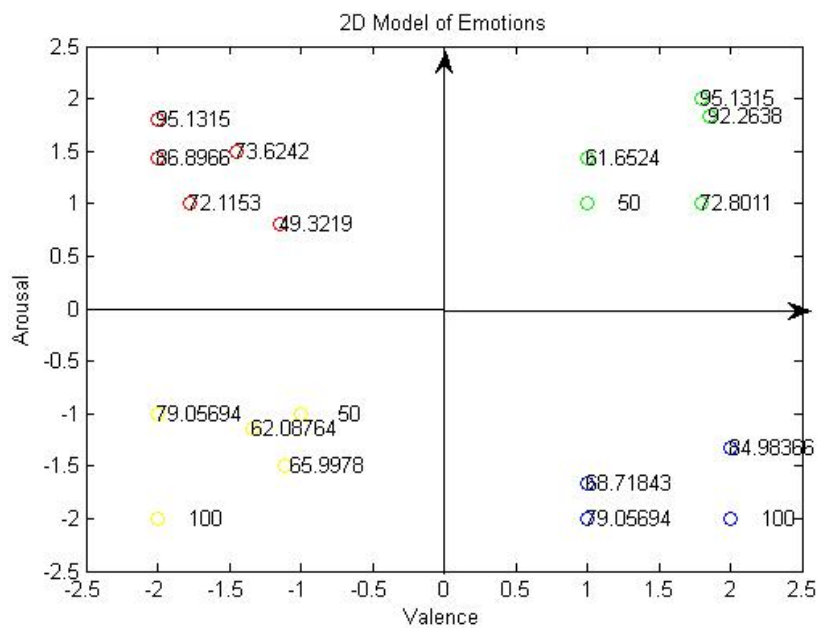

Figure 2. Labeling and quantification of emotions in 2D space of arousal and valence for 20 experiments. 
Table 2- List of extracted features from Poincare plot of heart rate series for 2D regression models of emotions (LOI: Line of Identity)

\begin{tabular}{|c|c|}
\hline Feature & Description \\
\hline SD1[10] & Standard Descriptor 1: $S D 1=\sqrt{\operatorname{var}\left(\frac{R R_{i}-R R_{i+1}}{\sqrt{2}}\right)}$ \\
\hline SD2 [10] & Standard Descriptor 2: $S D 2=\sqrt{\operatorname{var}\left(\frac{R R_{i}+R R_{i+1}}{\sqrt{2}}\right)}$ \\
\hline $\mathrm{Cn}[11]$ & The Area of the Fitted Ellipse: $C n=\pi \times S D 1 \times S D 2$ \\
\hline $\mathrm{CCM}[11]$ & Complex Correlation Measure: $C C M=\frac{1}{C n(n-2)} \sum_{i=1}^{n-2}\|A(i)\| ; A(i)=\frac{1}{2}\left|\begin{array}{ccc}R R_{i} & R R_{i+1} & 1 \\
R R_{i+1} & R R_{i+2} & 1 \\
R R_{i+2} & R R_{i+2} & 1\end{array}\right|$ \\
\hline Above [12] & Number of points which are above LOI in Poincare plot \\
\hline On [12] & Number of points which are on LOI in Poincare plot \\
\hline Below [12] & Number of points which are below LOI in Poincare plot \\
\hline Above-Above [12] & \multirow{2}{*}{$\begin{array}{l}\text { Number of consecutive points in which both of them are above the LOI in Poincare plot } \\
\text { Number of consecutive points in which the first is above and the second is on the LOI in Poincare plot }\end{array}$} \\
\hline Above-On [12] & \\
\hline Above-Below [12] & Number of consecutive points in which the first is above and the second is below the LOI in Poincare plot \\
\hline On-Above [12] & Number of consecutive points in which the first is on and the second is above the LOI in Poincare plot \\
\hline On-On [12] & \multirow{2}{*}{$\begin{array}{l}\text { Number of consecutive points in which both of them are on the LOI in Poincare plot } \\
\text { Number of consecutive points in which the first is on and the second is below the LOI in Poincare plot }\end{array}$} \\
\hline On-Below [12] & \\
\hline Below-Above [12] & Number of consecutive points in which the first is below and the second is above the LOI in Poincare plot \\
\hline Below-On [12] & Number of consecutive points in which the first is below and the second is on the LOI in Poincare plot \\
\hline Below-Below [12] & Number of consecutive points in which both of them are below the LOI in Poincare plot \\
\hline PI [13] & Porta Index; $P I=\frac{\text { Down }}{U p+\text { Down }} \times 100$ \\
\hline GI [14] & Guzik Index; $G I=\frac{\sum_{i=1}^{U p} D i^{+}}{\sum_{i=1}^{U p} D i^{+}+\sum_{i=1}^{D O w n} D i^{-}} \times 100$ \\
\hline SI [15] & Slope Index; $S I=\frac{\sum_{i=1}^{U p} \text { Relative Slope of Points Above the Line of Identity }}{\sum_{i=1}^{U p+D o w n} \text { Relative Slope of Points which are not On the Line of Identity }}$ \\
\hline$S D 1_{d}^{2}[16]$ & $S D 1_{d}^{2}=\frac{1}{n} \sum_{i=1}^{U p}\left[r_{i}\right]^{2}$ \\
\hline$S D 1_{a}^{2}[16]$ & $S D 1_{a}^{2}=\frac{1}{n} \sum_{i=1}^{\text {Down }}\left[r_{i}\right]^{2}$ \\
\hline$S D 2_{d}^{2}[16]$ & $S D 2_{d}^{2}=\frac{1}{2}\left[\sum_{i=1}^{U p}\left[r_{i}\right]^{2}+\frac{1}{2} \sum_{i=1}^{O n}\left[r_{i}\right]^{2}\right]$ \\
\hline$S D 2_{a}^{2}[16]$ & $S D 2_{a}^{2}=\frac{1}{2}\left[\sum_{i=1}^{D \text { own }}\left[r_{i}\right]^{2}+\frac{1}{2} \sum_{i=1}^{\text {On }}\left[r_{i}\right]^{2}\right]$ \\
\hline$S D N N_{d}^{2}[16]$ & $S D N N_{d}^{2}=\frac{1}{2}\left(S D 1_{d}^{2}+S D 2_{d}^{2}\right)$ \\
\hline$S D N N_{a}^{2}[16]$ & $S D N N_{a}^{2}=\frac{1}{2}\left(S D 1_{a}^{2}+S D 2_{a}^{2}\right)$ \\
\hline$C_{1 d}[16]$ & $C_{1 d}=\frac{S D 1_{d}^{2}}{S D 1^{2}}$ \\
\hline$C_{1 a}[16]$ & $C_{1 a}=\frac{S D 1_{a}^{2}}{S D 1^{2}}$ \\
\hline$C_{2 d}[16]$ & $C_{2 d}=\frac{S D 2_{d}^{2}}{S D 2^{2}}$ \\
\hline$C_{2 a}[16]$ & $C_{2 a}=\frac{S D 2_{a}^{2}}{S D 2^{2}}$ \\
\hline$C_{d}[16]$ & $C_{d}=\frac{S D N N_{d}^{2}}{S D N N^{2}}$ \\
\hline$C_{a}[16]$ & $C_{a}=\frac{S D N N_{a}^{2}}{S D N N^{2}}$ \\
\hline
\end{tabular}

\subsection{Evaluating the Results of Emotions Assessment}

The classification performance is shown in Table 4. To the best of our knowledge, there is no similar method or psychological technique for measuring the strength of emotions like what is proposed in this article that warrant future study to evaluate the usefulness and performance of proposed method for quantification of emotions. 
Table 3. Mean square error for estimation of the arousal and valence levels

\begin{tabular}{c|c} 
& Mean Square Error \\
\hline Arousal Tree-based Model & 0.0393 \\
\hline Valence Tree-based Model & 0.0536
\end{tabular}

Table 4. Classification results

\begin{tabular}{c|c|c|c} 
Emotions & $\begin{array}{c}\text { Accuracy } \\
\text { (\%) }\end{array}$ & $\begin{array}{c}\text { Sensitivity } \\
\text { (\%) }\end{array}$ & $\begin{array}{c}\text { Specificity } \\
\text { (\%) }\end{array}$ \\
\hline Pleasure & 97.5 & 94.28 & 98.57 \\
\hline Joy & 93.21 & 84.28 & 96.19 \\
\hline Anger & 93.92 & 90 & 95.23 \\
\hline Sadness & 98.21 & 97.14 & 98.57
\end{tabular}

\section{Conclusion}

In this article, the arousal and valence levels were estimated using 2D regression models of emotions for heart rate series recorded while inducing emotions by color stimuli. The obtained arousal and valence levels used in two phases: first for emotion detection and second for quantification of the strength of emotion. The proposed method for measuring emotions can be of great help to psychiatrists to identify mental disorders. Using this method, one can detect and express the strength of emotions individually and has a comparative criterion for emotions in different individuals.

\section{References}

[1] S. Moharreri, N. Jafarnia Dabanloo, S. Parvaneh, and A. M. Nasrabadi, "how to interpret psychology from heart rate variability?," in Middle East Conference on Biomedical Engineering (MECBME), Sharjah, UAE, Feb. 22-25 2011, vol. 1: IEEE.

[2] S. Moharreri, N. Jafarnia Dabanloo, S. Parvaneh, and A. M. Nasrabadi, "the relation between colors, emotions and heart response using triangle phase space mapping (TPSM)," presented at the Computing in Cardiology (CinC2011), 2011.

[3] K. H. Kim, S. W. Bang, and S. R. Kim, "emotion recognition system using short-term monitoring of physiological signals," Medical \& biological Engineering \& Computing, vol. 42, pp. 419-427, 2004.

[4] B. Hwang, J. W. Ryu, C. Park, and B. T. Zhang, "a novel method to monitor human stress states using ultra-short-term ecg spectral feature," in 39th Annual International Conference of the IEEE Engineering in Medicine and Biology Society (EMBC), Seogwipo, 2017, vol. 39: IEEE, pp. 23812384.

[5] K.-H. Choi, J. Kim, O. s. Kwon, M. J. Kim, Y. H. Ryu, and J.-E. Park, "is heart rate variability (hrv) an adequate tool for evaluating human emotions? - a focus on the use of the international affective picture system (IAPS)," Psychiatry Research, vol. 251, pp. 192-196, 2017.

[6] S. Rezaei, S. Moharreri, S. Ghiasi, and S. Parvaneh, "emotion recognition using parabolic phase space mapping for heart rate variability analysis," presented at the Computing in Cardiology (CinC), 2017.

[7] H.-J. Suk and H. Irtel, "emotional response to color across media," Color Research and Application, vol. 35, pp. 64-77, 2008.

[8] J. Pan and W. J. Tompkins, "a real-time qrs detection algorithm," Biomedical Engineering, IEEE Transactions on, no. 3, pp. 230-236, 1985.

[9] S. Moharreri, N. Jafarnia Dabanloo, and K. Maghooli, "modeling the $2 \mathrm{~d}$ space of emotions based on the poincare plot of heart rate variability signal," Biocybernetics and Biomedical Engineering, vol. 38, pp. 794-809, 2018. ELSEVIER.

[10] M. Brennan, M. Palaniswami, and P. Kamen, "do existing measures of poincare plot geometry reflect nonlinear features of heart rate variability?," IEEE Transactions on Biomedical Engineering, vol. 48, no. 11, pp. 1342-1347, 2001.

[11] C. K. Karmakar, A. H. Khandoker, J. Gubbi, and M. Palaniswami, "complex correlation measure: a novel descriptor for poincare plot," Biomedical Engineering OnLine, vol. 8, pp. 1-12, 2009.

[12] S. Moharreri, S. Parvaneh, N. Jafarnia Dabanloo, and A. M. Nasrabadi, "utilizing occurrence sequence of heart rate's phase space points in order to discriminate heart arrhythmia," in the 17th Iranian Conference of Biomedical Engineering (ICBME2010), Isfahan, Iran, 3-4 November 2010, vol. 17: IEEE.

[13] A. Porta et al., "temporal asymmetries of short-term heart period variability are linked to autonomic regulation," American Journal of Physiology-Regulatory, Integrative and Comparative Physiology, vol. 295, no. 2, pp. R550R557, 2008.

[14] J. Piskorski and P. Guzik, "geometry of the poincaré plot of rr intervals and its asymmetry in healthy adults," Physiological measurement, vol. 28, no. 3, p. 287, 2007.

[15] C. K. Karmakar, A. Khandoker, and M. Palaniswami, "analysis of slope based heart rate asymmetry using poincare plots," presented at the Computing in Cardiology (CinC), 2012.

[16] J. Piskorski and P. Guzik, "compensatory properties of heart rate asymmetry," Journal of electrocardiology, vol. 45, pp. 220-224, 2012.

Address for correspondence.

Saman Parvaneh

2 Canal Park, 3rd floor, Cambridge, MA 02141

parvaneh@ieee.org 